\title{
Abnormal Iron Accumulation in Brain and New Iron Chelator for Labile Iron Removal Therapy
}

\author{
Yuzo Nishida ${ }^{1}$ \\ ${ }^{1}$ Medical Research Institute, Kanazawa Medical University, Uchinada, 920-0293, Japan \\ Correspondence: Yuzo Nishida, Medical Research Institute, Kanazawa Medical University, Uchinada, 920-0293, \\ Japan. E-mail:nsd-2210@kanazawa-med.ac.jp
}

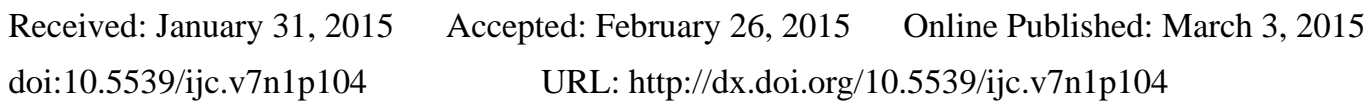

\begin{abstract}
The homeostasis of transition metal ions is central to many life processes, but the alterations in Fe, $\mathrm{Zn}$, and Mn homeostasis are observed in the brain of patients of several neurodegenerative disorders. Abnormally high levels of non-transferrin-bound iron ions (NTBI), or labile iron ion, have been demonstrated in a number of neurodegenerative disorders including dementia, Parkinson's disease (PD) and Alzheimer's disease (AD), and oxidative stress closely related with NTBI in the brain is widely believed to be associated with neuronal death in these diseases. We have investigated the chemical mechanism of the abnormal accumulation of iron ions in the brain observed for the patients of neurodegenerative disorders, and based on these results we have prepared the new iron chelators, so-called super-polyphenols. Since our super-polyphenols can excrete only NTBI effectively from the plasma, not the iron in the holo-transferrin, our super polyphenols should be one of the most hopeful substances for labile iron removal therapy, including the prevention of dementia, Alzheimer's and Parkinson's diseases. Our super-polyphenols are characterized by the following four points, i.e., 1) these super-polyphenols are water-insoluble, 2) these are not metabolized in the human body due to its insolubility in water and its polymeric structure (MW 90,000), 3) their iron (III) chelates are also water-insoluble, and 4) they do not interact with the iron ions in the holo-transferrin, and because of the these properties our super-polyphenols give no damage to human body.
\end{abstract}

Keywords: NTBI, hydrogen peroxide, oxidative stress, neurodegeneration, super-polyphenols

\section{Introduction}

Iron is utilized by several proteins as cofactor for major biological processes, however, iron may also harm cells by promoting oxidative stress. Plasma transferrin has been known for years as a central player in iron metabolism, assigned to circulate iron in a soluble, non-toxic form and deliver it to the erythron and other tissues, and it exerts a crucial role in the maintenance of systemic iron homeostasis (Gkouvatos, Papanikolauo \& Pantopoulos, 2012). When some chelates (amino acids derivatives, small peptides or citrate, etc.) are present in the plasma, the hemosiderin and iron deposition which contain polymeric iron (III) ions with oxo-bridges may dissolve; these water-soluble iron ions not associated with transferrin is generally termed as non-transferrin-bound iron (NTBI), or labile iron ion. NTBI is detected in the plasma of patients with thalassemia, hemochromatosis and other iron-overloading disorders, and is present at concentration up to $10 \mu \mathrm{M}$ (Hershko, Link, Konjin \& Cabantchik, 2005; Evans, et al. 2008).

Abnormally high levels of iron (NTBI) including iron deposition in the brain have been demonstrated in a number of neurodegenerative disorders including Parkinson's disease (PD) and Alzheimer's disease (AD), and oxidative stress closely related with the increased iron levels in the brain is widely believed to be associated with neuronal death in these diseases (Stankievicz, Panter, Neema, Aroma, Batt \& Bakshi, 2007; Gaeta \& Hider, 2005). Recently the origin of the iron toxicity to induce oxidative stress has been elucidated by us on the chemical point of view (Nishida, 2003, 2004, 2011, 2012a, 2012b); in our mechanism the contribution of hydroxyl radical is completely denied, which has been supported by the recent work by Enami et al. (Enami, Sakamoto \& Colussi, 2014); the formation of the $\mathrm{Fe}(\mathrm{IV})=\mathrm{O}$ species observed by Enami et al. can be reasonably explained in terms of concerted mechanism proposed by Nishida (Nishida, 2012b). In addition to this, we have succeeded in elucidating the mechanism of accumulation of iron ion and formation of iron deposition in the brain of patients with neurodegenerative disorders (Nishida, 2012c, Abe, Sakiyama \& Nishida, 2015a). 
For the prevention and treatment of many diseases related with NTBI, especially dementia, Alzheimer's and Parkinson's diseases, we have prepared new iron chelators which remove only NTBI from the plasma effectively and without toxicity; these chelates are called as super-polyphenols(Nishida, 2012a; Nishida, Kohgo, Sasaki \& Ikuta, 2012), and its characterization will be developed briefly in this article.

\section{Role of Hydrogen Peroxide on Oxidative Stress Due to NTBI}

\subsection{Hydrogen Peroxide and NTBI}

We already reported that hydrogen peroxide promotes the formation of di- $\mu$-oxo-diiron(III) species (species (B) in Scheme I) from several iron(III) chelates with amino acid derivative in the presence of reducing agents such as alcohol or phenol (see Scheme I) (Sutoh, Okawakumai \& Nishida, 2006); as seen in the Figure 1, the formation of di- $\mu$-oxo-diiron(III) species, $\mathrm{Fe}_{2}(\mathrm{O})_{2}(\mathrm{dpal})_{2}$ due to the addition of $\mathrm{H}_{2}{ }^{16} \mathrm{O}_{2}$ can be confirmed by the peak at $\mathrm{m} / \mathrm{z}=683.2$.

\section{Scheme I}

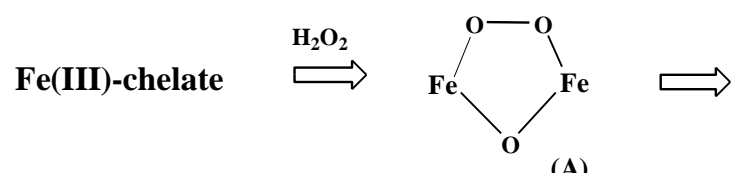

(A)

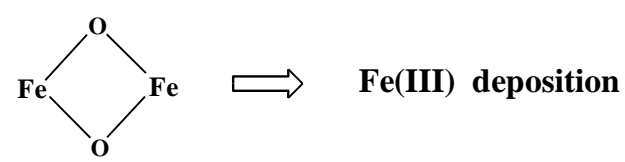

(C)

(B)
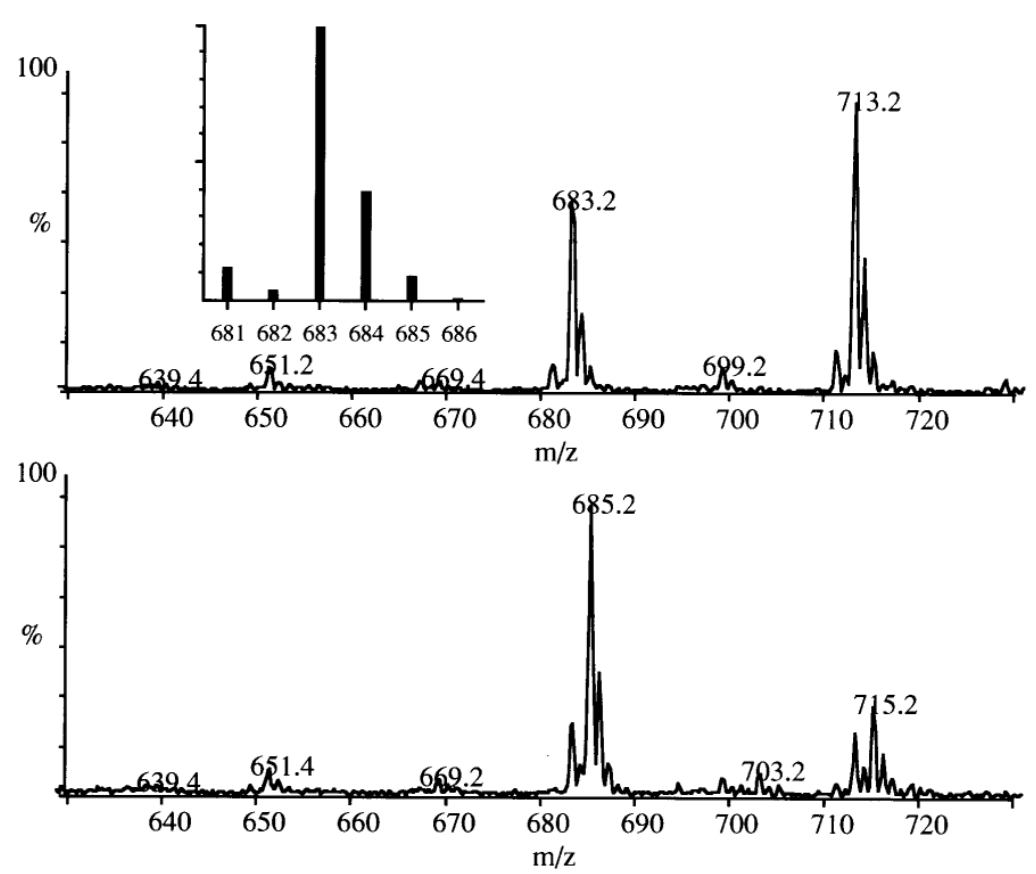

Figure 1. Mass spectral data of the solution (water/methanol) containing $\left[\mathrm{Fe}(\mathrm{III})(\mathrm{dpal}) \mathrm{Cl}_{2}\right]$ and hydrogen peroxide, where $\mathrm{H}(\mathrm{dpal})$ represents $N, N$-bis(2- pyridylmethyl)- $\beta$-alanine. Upper: $\mathrm{H}_{2}{ }^{16} \mathrm{O}_{2}$ was added in the solution. Lower: $\mathrm{H}_{2}{ }^{18} \mathrm{O}_{2}$ was added in the solution.

When the $\mathrm{H}_{2}{ }^{18} \mathrm{O}_{2}$ was added instead of $\mathrm{H}_{2}{ }^{16} \mathrm{O}_{2}$ the peak at 683.2 in the upper figure shifts to 685.2 in the lower, clearly demonstrates that one of the oxo-atom in the di- $\mu$-oxo-diiron(III) species is derived from the hydrogen peroxide used (Sutoh, Okawakumai \& Nishida, 2006)]. This should be due to the strong electrophilicity of the ( $\mu$-peroxo)( $\mu$-oxo)-diiron(III) species (species (A) in Scheme I) formed in the solution (Nishida, 2003, 2004, 2011, 2012a, 2012b), which turns to the di- $\mu$-oxo-diiron(III) species by oxidizing the methanol in the solution The further aggregation of the di- $\mu$-oxo-diiron(III) species may proceed to give the iron deposition (see (C) in 
Scheme I), because it has been pointed out that the iron deposition is the aggregation of di- $\mu$-oxo-diiron (III) species based on the structural properties of hydroxo(oxo)iron clusters(Nishida, 2012a, 2012c); this is exemplified by the recent our work (Abe, Sakiyama \& Nishida, 2015a). As these polymeric iron(III) ions are not transferred to apo-transferrin(Nishida, Ito \& Satoh, 2007; Nishida, 2012c), above discussion explains the marked iron accumulation in the brain as well as visceral tissue despite low serum iron levels, and under these conditions where excess hydrogen peroxide is present. It seems quite likely that highly toxic ( $\mu$-peroxo)diiron(III) species ((A) in Scheme I) generates in a facile manner, degrading the peripheral proteins or DNA, inducing severe oxidative damage, which are all consistent with those observed in the aceruroplasminemia patients (Yoshida, et al. 2000), where in the patient of aceruroplasminemia, $\mathrm{Fe}^{2+}$ ions are oxidized by apo-transferrin to $\mathrm{Fe}^{3+}$ ions with the formation of hydrogen peroxide (Nishida, 2012c). Similar ferroxidase-like function was also observed for APP, amyloid precursor protein (Duce, et al. 2011), and thus promoted production of APP should be closely related with the increase of AD patients as observed (Roberts, et al. 2012).

This means that oxidative stress already proceeds in the places where iron deposition is observed, and this should be a clear answer for the questions demonstrated in reference ((Stankievicz, Panter, Neema, Aroma, Batt \& Bakshi, 2007)

\subsection{Disorders Due to Abnormal Ferritin}

Mammalian ferritins are heteropolymers made of two different subunit types named $\mathrm{H}-$ and L-chain with equivalent proportion. The $\mathrm{H}$-chain carries a ferroxoidase center which appears to be essential for iron corporation, whereas the L-chain facilitates iron mineralization inside the cavity (see Equation (1) below) (Harrison \& Arosio, 1996); Chasteen \& Harrison, 1999)

\section{H-chain}

$$
\begin{aligned}
& \text { Protein }+2 \mathrm{Fe}^{2+}+\mathrm{O}_{2}+3 \mathrm{H}_{2} \mathrm{O} \quad \longrightarrow \\
& \text { Protein- }\left[\mathrm{Fe}_{2}(\mathrm{O})(\mathrm{OH})_{2}\right]^{2+}+\mathrm{H}_{2} \mathrm{O}_{2}+2 \mathrm{H}^{+}
\end{aligned}
$$

\section{L-chain}

$$
\begin{aligned}
& \text { Protein- }\left[\mathrm{Fe}_{2}(\mathrm{O})(\mathrm{OH})_{2}\right]^{2+} \\
& \text { Protein }+2 \mathrm{FeOOH}(\text { core })+2 \mathrm{H}^{+}
\end{aligned}
$$

\section{Equation (1)}

It is well known that in the H-chain, two iron(II) ions are oxidized to an iron(III) state through the formation of ( $\mu$-peroxo)diiron(III) species ((A) in Scheme I); this process is almost the same as observed for the reactions in RNR (ribonucleotide reductase) (Anderson, Hogborn, R.-Mathis, Anderson, Sjoberg \& Nortlund, 1999) and MMO (methane monooxygenase) (Baik, Newcome, Friesner \& Lippard, 2003). Although the hydrogen peroxide generated is used for the oxidation reactions of the organic compounds in RNR and MMO, the hydrogen peroxide formed in the H-chain is only released from the site! This difference of the reactivity of peroxide ion in the ( $\mu$-peroxo)diiron(III) species among the enzymes has been elucidated by us (Nishida, 2012b, Nishida, Itoh \& Okuno, 1997, Nishino, Takahashi \& Nishida, 2002, Nishida, 1998) i.e., in the RNR and MMO the peripheral carboxylate group contributes to activate the peroxide ion through the formation of the peroxide adduct, as shown in Figure 2, but the hydrogen peroxide formed in H-chain is released from the site because of the absence of peripheral glutamate.

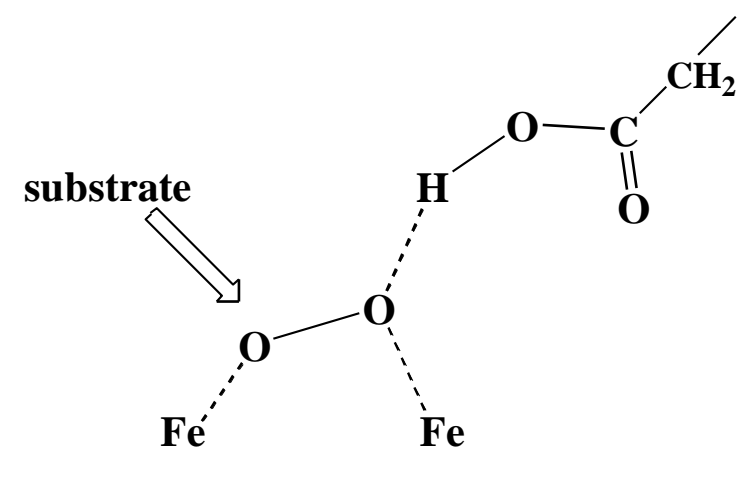

Figure 2. Assumed intermediate for MMO [8,21,23]. 
We have demonstrate (Sutoh, Okawakumai \& Nishida, 2006) that the hydrogen peroxide released from H-chain is necessary in the L-chain for the mineralization of the iron cores through the forming a $\mathrm{Fe}$-oxo bonding as illustrated in Scheme I, although the role of hydrogen peroxide in the L-chain has been completely neglected in the previous papers as illustrated in Equation (1) (Harrison \& Arosio, 1996); Chasteen a\& Harrison, 1999). We also demonstrated that rich carboxyl groups exposed on the active surface of L chain is necessary for the normal iron nucleation and mineralization on the basis of results on the crystal structural determination of several iron(III) compounds (Sutoh, Okawakumai \& Nishida, 2006), and have pointed out that the precise formation of heteropolymers by the $\mathrm{H}$ - and L-chains with equivalent proportion is essential for the utilizing of the peroxide ion; this is the main reason for that ferritin is non-toxic.

Although H- and L-chains are encoded by different genes, synthesis of both chains is controlled by a common cytosolic protein, iron regulatory protein (IRP), which binds to the iron responsive-element (IRE) in the 5'-UTR of the $\mathrm{H}$ - and L-chain mRNAs. Kato et al. showed that a single mutation in the IRE region of $\mathrm{H}$-chain mRNA induces the decrease of H-chain, and affected dominantly inherited iron overload, leading to tissue iron deposition, and also to impairment of the ferroxidase activity by H-chain (Kato, et al., 2001) Hereditary hyperferritinemia-cataract syndrome (HHCS) is an autosomal and dominant disease caused by heterogeneous mutations in the IRE of the 5'-untraslated flanking region of ferritin L-chain mRNA. According to the results by Levi et al., L-chain levels are up to 20-fold higher in HHCS than in control cells, and were not affected by iron supplementation or chelation (Levi, et al., 1998). Above facts are implying that mutations in mRNAs induce the unbalance between $\mathrm{H}$ - and $\mathrm{L}$-chains, leading to the abnormal presence of hydrogen peroxide in the ferritin, which leads to the formation of iron deposition as illustrated in Scheme I, and also to the enhanced toxicity due to hydrogen peroxide and NTBI as demonstrated by us (Nishida, 2004; 2012b).

Neuroferritinopathy typically presents with progressive adult-onset chorea or dystonia affecting one or two limbs, and subtle cognitive deficits (Ohta \& Takiyama, 2012). The movement disorder involves additional limbs within five to ten years and becomes more generalized within 20 years. The majority of individuals develop a characteristic orofacial action-specific dystonia related to speech that leads to dysarthrophonia. Cognitive deficits with time. Neuroferritinopathy is a neurodegenerative disease which demonstrates brain iron accumulation caused by the mutations in the ferritin L-chain gene (Curtis, et al., 2001) Thus, it seems quite reasonable to assume that the presence of excess iron accumulation in the patients should be due to the disruption of equivalency between the $\mathrm{H}$ - and $\mathrm{L}$-chains in the ferritin, as pointed out for hereditary hyperferritinemia-cataract syndrome (HHCS).

\subsection{Hydrogen Peroxide in the Patients of $P D$ and $A D$}

Iron accumulates as the brain ages and has been linked to motor and cognitive dysfunction in the elderly, and a growing body of data suggests that brain iron accumulation in vivo contribute to tissue damage in a variety of chronic neurologic disorders; these are consistent with the histologic and MRI data (Stankievicz, Panter, Neema, Aroma, Batt \& Bakshi, 2007). In AD, lipofuscin may play a role in the modulation of the release or iron from damaged mitochondoria, which becomes as important generator of $\mathrm{H}_{2} \mathrm{O}_{2}$. The formation of $\mathrm{H}_{2} \mathrm{O}_{2}$ should occur through a binuclear iron(III) chelate formation as pointed out by Nishida (Nishida, 2004, 2011, 2012a, 2012b), and the observed tissue damage can be elucidated in terms of Scheme I. The fact that increasing of pathogenesis of $\mathrm{AD}$ is induced by ferritin abnormality (Roberts, et al. 2012) can be elucidated by the increased $\mathrm{H}_{2} \mathrm{O}_{2}$ formation from the abnormal ferritin as described above, which gives serious oxidative stress in the presence of NTBI as shown in Scheme I.

The following phenomena have all been observed in the Parkinsonian brains: (a) decline in the mitochondoria activity, (b) generation of $\mathrm{H}_{2} \mathrm{O}_{2}$, following the deamination of dopamine by monoamineoxidase and by autooxidation (this should occur through the catalytic function by water-soluble NTBI), (c) increased activity of superoxide dismutase, (d) reduced concentration of glutathione, and (e) elevated level of iron in microglia, astrocytes, oligodendrocytes, and dopaminergic neurons of SNc (Gaeta \& Hider, 2005). These data clearly show that high level of $\mathrm{H}_{2} \mathrm{O}_{2}$ are present in the brain of both the patients of $\mathrm{PD}$ and $\mathrm{AD}$, and these $\mathrm{H}_{2} \mathrm{O}_{2}$ may lead to the formation of polymeric iron(III) compounds which are not transferred to transferrin (Nishida, Ito \& Satoh, 2007), and also act as an active oxidant towards proteins or DNA through the formation of ( $\mu$-peroxo)-diiron(III) species (A) shown in Scheme I.

\section{New Chelating Agents to Remove NTBI from the Plasma}

As shown in our previous papers (Nishida, 2004, 2012a, 2012b; Abe, Sakiyama \& Nishida, 2015b), the formation of $\mathrm{H}_{2} \mathrm{O}_{2}$ is greatly promoted by NTBI. For the removal of NTBI the iron chelation method has been used, but almost all the chelates used until now are less effective or have certain adverse effects (Agarrwal, 
2006). A new synthetic oral iron chelator, Exjade, is high effective (Cappellini, 2007), but serious side effects are also reported for Exjade in NO.270 Report from The Ministry of Health, Labour and Welfare of Japan (2010).

Very recently we have prepared the so-called super-polyphenols which contain more than 100 molecules of catechol derivative in one polymeric compound, chitosan (Nishida, 2012a, Nishida, Kohgo, Sasaki \& Ikuta, 2012) (see Figure 3). We found that water-insoluble super-polyphenols can eliminate NTBI effectively in vitro study, as exemplified in Figure 4. Our super-polyphenols are characterized in the following four points, 1) the super-polyphenols are water-insoluble, 2) these are not metabolized in the human body due to its insolubility in water and its polymeric structure (MW 90,000), 3) their iron (III) chelates are also water-insoluble, and 4) they do not interact with the iron ions in the holo-transferrin. The property 4) clearly indicates that our super-polyphenols can discriminate the necessary iron ion and unnecessary iron ion (NTBI) in the human body, and can remove only the NTBI from the body.

Over the past decade, research with curcumin has increased significantly (Balkacemi, Doggui, Dao \& Ramassamy, 2011). In vitro and in vivo studies have demonstrated that curcumin could target pathways involved in the pathophysiology of AD. These findings suggest that curcumin might be a promising compound for the development of AD therapy, but its insolubility in water and poor bioavailability have limited clinical trials and its therapeutic applications. We have found that the water-insoluble super-polyphenol containing vanillin derivative shows high ability to catch NTBI through chelation, implying that the antioxidant, anti-inflammatory, antiproliferative properties of curcumin should be due to its ability to excrete NTBI, and thus it seems quite likely that our super-polyphenols can be one of the most hopeful substances for labile iron removal therapy, including the prevention of dementia, Alzheimer's and Parkinson's diseases, and thus I hope that our super-polyphenols will be used over the whole world in future for the prevention and treatment of many diseases related with NTBI, and this project is now under progress in Japan.

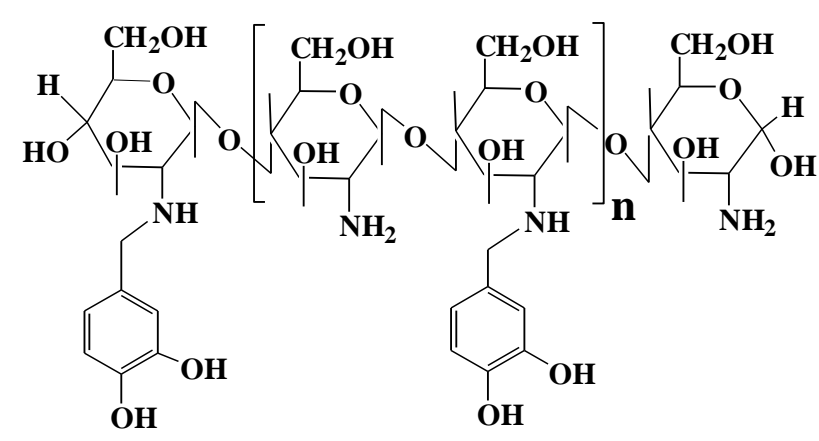

Figure 3. An example of super-polyphenols, FC-Cate2.

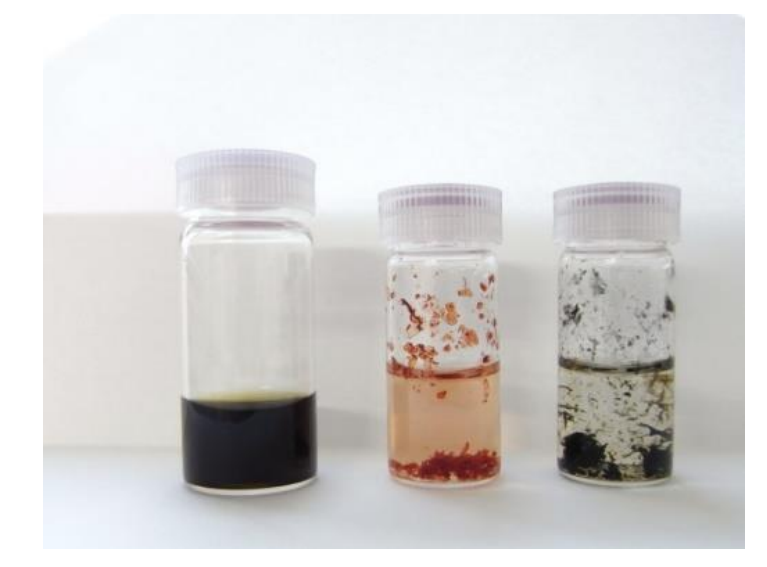

Figure 4. Left: solution of Fe(III)-(nta) chelate (Nishida \& Ito, 1995)

Center: Super-polyphenol, FC-Carb2 (solid) was added to the Fe(III)-(nta) solution

Right: Super-polyphenol, FC-Cate2 (solid) was added to the Fe(III)-(nta) solution 


\section{References}

Abe, K., Sakiyama, H., \& Nishida, Y. (2015a). Model reactions for the formation of Iron Deposition. Int. J. Chem., 7(1), 21-27.

Abe, K., Sakiyama, H., \& Nishida, Y. (2015b). New Insight into Toxicity due to Oligomeric Amyloid- $\beta$ Peptide and Alpha-Synuclein Protein induced by Copper(II) ion. Int. J. Chem., 7(1), 41-47.

Agarwal, M. B. (2006). Exjade: A new oral iron chelator. JAPI, 54, 214-217.

Andersson, M. E, Hogbom, M., R-Matthis A., Andersson, K. K., Sjoberg, B., \& Nortlund, P. (1999). The crystal structure of an azide complex of the differous R2 subunit of ribonucleotide reductase displays a novel carboxylate shift with important mechanistic implication for diiron-catalayzed oxygen activation. J. Am. Chem. Soc. 121, 2346-2352.

Baik, M., Newcome, M., Friesner, R. A., \& Lippard, S. J. (2003). Mechanistic studies on the hydroxylation of methane by methane monooxygenase. Chem. Rev., 103, 2385-2419. http://dx.doi.org/10.1021/cr950244f

Belkacemi, A., Doggui, S., Dao, L., \& Ramassamy, C. (2011). Challenges associated with curcumin therapy in Alzheimer's disease. Expert. Rev. Mol. Med., 4, 13-20. http://dx.doi.org/10.1017/S1462399411002055.

Cappellini, M. D. (2007). Exjade in the treatment of chronic iron overload associated with blood transfusion. Ther. Clin. Risk Manag., 3, 291-299.

Chasteen, N. D., \& Harrison, P. M. (1999). Mineralization in ferritin: an efficient means of iron storage. J. Struc. Biology, 126, 182-194.

Curtis, A. R., Fey, C., Morris, C. M., Bind, L. A., Patrick, P. G., Chinnery, P. F., .. Burn, J. (2001). Mutation in the gene region encoding basal ganglia disease. Nat. Genet., 28, 350-354. http://dx.doi.org/10.1038/ng571

Duce, J. A., Tsatsanis, A., Cater, M. A., James, S. A., Robb, E., Wikhe, K., ... Bush, A. I. (2010). An iron-export ferroxidase activity of $\beta$-amyloid protein precursor is inhibited by zinc in Alzheimer's disease. Cell, 142, 857-867. http://dx.doi.org/10.1016/j.cell.2010.08.014.

Enami, S., Sakamoto, Y., \& Collusi, A. J. (2014). Fenton chemistry at aqueous interfaces. Proc. Natl. Acad. Sci. USA., 111, 623-629. http://dx.doi.org/10.1073/pnas.1314885111.

Evans, R. W., Rafique, R., Zarea, A., Rapisarda, C., Cammack, R., Evans, P. J., Porter, J. B., \& Hider, R. C. (2008) Nature of non-transferrin-bound iron: studies on iron citrate complexes and thalassemia sera. J. Biol. Inorg. Chem. 13, 57-74. http://dx.doi.org/10.1007/s00775-007-0297-8

Gaeta, A., \& Hider R. C. (2005). The crucial role of metal ions in neurodegeneration: the basis for a promising therapeutic strategy. Brit. J. Pharmacology, 146, 1041-1059. http://dx.doi.org/10.1038/sj.bip.0706416

Gkouvatsos. K., Papanikolauo, G., \& Pantopoulos, K. (2012). Regulation of iron transport and the role of transferrin. Biochim Biophys Acta, 1820, 188-202. http://dx.doi.org/10.1016/j.bbagen.2011.10.013

Hershko, C., Link, G., Konjin, A., \& Cabantchik, Z. I. (2005) Objectives and mechanism of iron chelation therapy. Ann. N. Y. Acd. Sci., 1054, 124-135. http://dx.doi.org/10.1196/annals.1345.015

Harrison, P. M., \& Arosio, P. (1996). The ferritin: molecular properties, iron storage function and cellular regulation. Biochim. Biophys. Acta, 1275, 161-203.

Kato, J., Fujikawa, K., Kanda, M., Fukuda, N., Sasaki, K., Takayama, T., Kobune, K., Takada, K., Takimoto, R., Hamada, H., Ikeda, T., \& Niitsu, Y. (2001). A mutation, in the iron-responsive element of H-ferritin mRNA, causing autosomal dominant iron overload. Am. J. Hum. Genet., 69, 191-197.

Levi, S., Girelli, D., Perrone, F., Pasti, M., Beaumont, C., Corrocher, R., Albertini, A., \& Arosio, P. (1998) Analysis of ferritins in lymphoblastoid cell lines and in the lens of subjects with hereditary hyperferritinemia-cataract syndrome. Blood, 91, 4180-4187.

Nishida, Y. (1998). Important role of substrate in activation of dioxygen in biological oxygenases. Trends Inorg. Chem., 5, 89-103.

Nishida, Y. (2003). Elucidation of endemic neurodegenerative disorders. Z. Naturforsch., 58c, 752-758. http://www.znaturforsch.com/ac/v58c/s58c0752.pdf

Nishida, Y. (2004). Oxidative stress and neurodegeneration. Med. Hypothesis Res., 1, 227-245. http://www.journal-mhr.com/ PDF_Files/vol_1_4/1_4PDFs/1_4_2.pdf 
Nishida, Y. (2011). The chemical process of oxidative stress by copper(II) and iron(III) ions in several neurodegenerative disorders. Monatsh. Chem., 142, 375-384. http://dx.doi.org/10.1007/s00706-010-0444-8

Nishida, Y. (2012a). The chemical mechanism of oxidative stress due to non- transferrin- bound iron (NTBI). Adv. Biosci. Biotech., 3, 1076-1086. http://dx.doi.org/10.4236/abb.2012.327131.

Nishida, Y. (2012b). "Oxygen Activation, Oxidative Stress and Human Health", LAP Lambert Academic Publishing, Saarbrucken, Germany (2012).

Nishida, Y. (2012c). Role of zinc(II) ion for the formation of iron deposition in human body and its significance. Int. J. Chem., 4(6), 1-7. http://dx.doi.org/10.5539/ijc.v4n6p1

Nishida, Y., \& Ito, S. (1995). Structures and reactivity of several iron(III) complexes in the presence of hydrogen peroxide: relevance to induction of tissue damage caused by iron(III) chelates in rats. Polyhedron, 14, 2301-2308.

Nishida, Y., Ito, S., Okuno, T., \& Ohba, S. (1997). New insight into reaction of iron(III)-peroxide adduct with alkanes: an alternative model for cytochrome P-450 and methane monooxygenase. Z. Naturforsch, 52C, 615-622.

Nishida, Y., Itoh, Y., \& Satoh, T. (2007). Origin of renal proximal tubular injuries by Fe(III)-nta chelate. Z. Naturforsch, 62c, 608-612. http://www.znaturforsch.com/ac/v62c/s62c0608.pdf

Nishida, Y., Kohgo, Y., Sasaki, K., \& Ikuta, K. (2012). WO/2012/096183.

Ohta, E., \& Takiyama, Y. (2012). MRI findings in neuroferritinopathy. Neurology Research International 2012: 1-7. http://dx.doi.org/10.1155/2012/197438.

Roberts, B. R., Ryan, T. M., Bush, A. I., Masters, C. L., \& Duce, J. A. (2012). The role of metallobiology and amyloid- $\beta$ peptides in Alzheimer's disease. J. Neurochemistry, 120, 149-166.

Sutoh, Y., Okawamukai, Y., Nishino, S., \& Nishida, Y. (2006). Structure of a new tetranuclear iron(III) complex with an oxo-bridge; Factors to govern formation and stability of oxo-bridged iron(III) species in the L-subunit of ferritin. Z. Naturforsch, 61c, 149-154. http://www.znaturforsch.com/ac/v61c/s61c0149.pdf

Stankiewicz, J., Panter, S., Neema, M., Arora, A., Batt, C. E., \& Bakshi, R. (2007). Iron in chronic brain disorders: Imaging and neurotherapeutic implications. Neurotherapeutics, 4, 371-386. http://dx.doi.org/10.1016/j.nurt.2007.05.006

Yoshida, K., Kaneko, K., Miyajima, H., Tokuda, T., Nakamura, A., Kato, M., \& Ikeda, S. (2000). Increased lipid peroxidation in the brains of aceruplasminemia patients. J. Neurological Sci., 175, 91-95. http://dx.doi.org/10.1016/S0022-510X(00)00295-1

\section{Copyrights}

Copyright for this article is retained by the author(s), with first publication rights granted to the journal.

This is an open-access article distributed under the terms and conditions of the Creative Commons Attribution license (http://creativecommons.org/licenses/by/3.0/). 